\title{
Cerebral Hemodynamics in Patients with Rheumatoid Arthritis
}

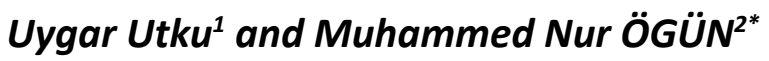

${ }^{1}$ Faculty of Medicine, Department of Neurology, istinye University, Turkey

${ }^{2}$ Faculty of Medicine, Department of Neurology, Abant Izzet Baysal University, Turkey

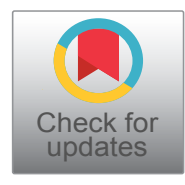

*Corresponding author: Muhammed Nur ÖGÜN, Assistant Professor, Faculty of Medicine, Department of Neurology, Abant Izzet Baysal University, 14280, Bolu, Turkey, Tel: +903742534656

\begin{abstract}
Objectives: The aim of this study was to assess the cerebral blood flow velocity using transcranial Doppler (TCD) ultrasonography in patients with Rheumatoid Arthritis (RA).

Materials and methods: A total of 22 patients aged 20 to 40-years-old with RA were enrolled in the RA group consecutively. Control group (non-RA group) consisted of 22 age and sex-matched, randomly selected patients without RA who had other diagnosis such as fibromyalgia and did not have risk factors for atherosclerosis (AS). Bilateral middle cerebral artery (MCA) peak-systolic, end-diastolic, and mean blood flow velocities, Pourcelot's resistance index values and Gosling's pulsatility index values were recorded with TCD by a neurosonologist blinded to RA and control groups.
\end{abstract}

Results: There were 22 people in each group. There were 10 women and 12 men in RA group. Therefore, control group was selected similar. The mean age was 38.6 in the RA group and 37.8 in the control group. Cerebral blood flow velocities of bilateral MCA were significantly higher in RA group than the control group.

Conclusions: Our study highlights the increased cerebral blood flow is indirectly associated with AS regarding persistent inflammation in patients with RA.

\section{Keywords}

Rheumatoid arthritis, Inflammation, Atherosclerosis, Stroke

\section{Introduction}

Nowadays, Rheumatoid Arthritis (RA) is known to be associated with cardiovascular diseases. However, the subject of risk factor that rheumatoid arthritis for cerebrovascular diseases is controversial. There has been recently considerable attention about the possible role of systemic inflammation in the development of atherosclerosis (AS) in patients with rheumatic diseases [1]. Increased risk of AS regarding cardiovascular events such as myocardial infarction and stroke leads to a significantly increased mortality in patients with RA $[2,3]$. The aim of this study was to assess the cerebral blood flow velocity using transcranial Doppler (TCD) ultrasonography in patients with RA.

\section{Patients and Methods}

A total of 22 consecutively admitted patients aged 20 to 50-year-old with a principal diagnosis of RA. The non-RA control group consisted of 22 age- and sexmatched, randomly selected patients who not have any risk factors for AS such as diabetes, hypertension, and coronary artery disease. The erythrocyte sedimentation rate (ESR), C-reactive protein (CRP) levels were measured to show the existence of inflammation. Consent was obtained from the local ethics committee, and all participants gave formal consent.

Examination of TCD ultrasonography was performed with Multi-Dop X DWL, TCD machine after the subjects rested in a supine position for $10 \mathrm{~min}$. The right and the left MCA were insonated from the temporal windows at 50-65 mm depth three times (for different depths) with $2 \mathrm{MHz}$ pulsed Doppler probes. Bilateral middle cerebral artery (MCA) peak-systolic, end-diastolic, and mean blood flow velocities, Pourcelot's resistance index (RI) values and Gosling's pulsatility index (PI) values were recorded with TCD by a neurosonologist blinded to RA and control groups. The results of RA group compared with the results of non-RA control group.

\section{Statistical Analysis}

The data was analyzed using the Statistical Package for Social Sciences (SPSS Inc, Chicago, IL, USA) 15.0 program. Paired t-test, cross-tabs tests were used for statistical analysis, $\mathrm{P}<0.05$ was considered significant.

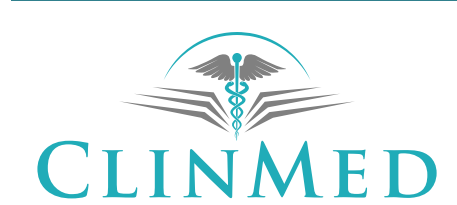

INTERNATIONAL LIBRARY

Citation: Utku U, ÖGÜN MN (2018) Cerebral Hemodynamics in Patients with Rheumatoid Arthritis. Int J Neurol Neurother 5:078. doi.org/10.23937/2378-3001/1410078

Accepted: December 13, 2018: Published: December 15, 2018

Copyright: (C) 2018 Utku U, et al. This is an open-access article distributed under the terms of the Creative Commons Attribution License, which permits unrestricted use, distribution, and reproduction in any medium, provided the original author and source are credited. 
Table 1: TCD data of RA group compared with control group.

\begin{tabular}{|c|c|c|c|}
\hline & RA group (n:22) & Control group (n:22) & $\mathbf{p}$ \\
\hline L-peak-systolic BFV & $140.2 \pm 12.4$ & $92 \pm 6.6$ & $<0.001$ \\
\hline L-end-diastolic BFV & $66.8 \pm 6.78$ & $33.4 \pm 2.15$ & $<0.001$ \\
\hline L-mean BFV & $106 \pm 5.45$ & $54.8 \pm 4.23$ & $<0.001$ \\
\hline L-PI & $0.87 \pm 0.09$ & $0.83 \pm 0.06$ & 0.22 \\
\hline L-RI & $06 \pm 0.05$ & $0.59 \pm 0.02$ & 0.33 \\
\hline R-peak-systolic BFV & $138.3 \pm 15$ & $94.3 \pm 11$ & $<0.001$ \\
\hline R-end-diastolic BFV & $69 \pm 10.98$ & $30 \pm 10$ & $<0.001$ \\
\hline R-mean BFV & $101.6 \pm 13.3$ & $58.9 \pm 9.34$ & $<0.001$ \\
\hline R-PI & $0.87 \pm 0.08$ & $0.83 \pm 0.09$ & 0.28 \\
\hline R-RI & $0.61 \pm 0.05$ & $0.56 \pm 0.04$ & 0.07 \\
\hline
\end{tabular}

L: Left, R: Right, PI: Pulsatility Index, RI: Resistance Index, BFV: Blood Flow Velocity.

\section{Results}

In this study $m / f: 12 / 10$, mean age: 37.8 was in RA group and $m / f: 12 / 10$, mean age: $35.2 \pm 4.9$ was in nonRA control group. Mean value of CRP and ESR levels were $12.5 \mathrm{mg} / \mathrm{L}(0-5 \mathrm{mg} / \mathrm{L})$ and $25.5 \mathrm{~mm} / \mathrm{h}$ respectively in $\mathrm{RA}$ group. There was a statistically significant difference between two groups for ESR and CRP levels. ESR and CRP levels were higher in RA group than the non-RA control group. Peak-systolic, end-diastolic, and mean blood flow velocities of bilateral MCA were significantly higher in RA group than the control group (Table 1). The $\mathrm{PI}$ and $\mathrm{RI}$ values were not significantly different in each group.

\section{Discussion}

The occurrence of nervous system manifestations in RA is rare. Inflammation plays an important role in the pathogenesis and progression of AS in patients with RA [4]. In RA group we found the mean values of ESR and CRP were higher than normal range. RA patients have higher levels of inflammatory markers, such as interleukin 6, tumor necrosis factor alpha and CRP [5].

Rheumatic patients have been reported to show early features of AS such as an increase in intimae media thickness in the carotid arteries and arterial stiffness [6]. Carotid-femoral pulse wave velocity was used as an indicator of arterial stiffness, whereas intimae media thickness in the carotid arteries was used to evaluate preclinical atherosclerosis [6]. We attempted to evaluate the cerebral blood flow velocities with using TCD ultrasonography in patients with RA because increased cerebral blood flow velocities are indirectly indicates an increase in AS.

We found peak-systolic, end-diastolic and mean blood flow velocities were significantly higher in RA group than the non-RA control group. But PI and RI values were not significantly different in each group. However, correct interpretation of $\mathrm{PI}$, because it depends not only on cerebrovascular resistance, but also on several systemic and cerebral variables [7]. The mechanisms that underlie this condition (increased velocity but normal PI values) are most likely to be due to mild diffuse AS or low disease activity is not associated with accelerated AS [8]. The Rotterdam study reported that increased mild to moderate cerebral blood flow velocity is due to diffuse AS or vasoconstriction [9]. This study had methodological limitations, including inadequate sample size and evaluation of activity and damage like joint restriction.

Our study highlights the increased cerebral blood flow is indirectly associated with AS regarding persistent inflammation in patients with RA.

\section{References}

1. Libby P, Ridker PM, Maseri A (2002) Inflammation and atherosclerosis. Circulation 105: 1135-1143.

2. Tejera-Segura B, de Vera-Gonzalez AM, Lopez-Mejias R, Gonzalez-Gay MA, Ferraz-Amaro I (2016) Serum cathepsin S and cystatin C: relationship to subclinical carotid atherosclerosis in rheumatoid arthritis. Clin Exp Rheumatol 30: 230-235.

3. Chia-Wei Lin, Ya-Ping Huang, Yueh-Hsia Chiu, Yu-Tsun Ho, Shin-Liang Pan (2014) Increased Risk of Ischemic Stroke in Young Patients with Ankylosing Spondylitis: A Population-Based Longitudinal Follow-Up Study. PLoS One 9: e94027.

4. Szekanecz Z, Kerekes G, Vegh E, Kardos Z, Barath Z, et al. (2016) Autoimmune atherosclerosis in 3D: How it develops, how to diagnosis and what to do. Autoimmun Rev 15: 756769.

5. Dessin PH, Corrales A, Lopez-Mejias R, Solomon A, Woodiwiss AJ, et al. (2016) The Framingham Score and the systematic coronary risk evaluation at low cut off values are useful surrogate markers of high-risk subclinical atherosclerosis in patients with rheumatoid arthritis. J Rheumatol 43: 486-494.

6. Ozen G, Inanc N, Unal AU, Korkmaz F, Sunbul M, et al. (2016) Subclinical atherosclerosis in systemic sclerosis: not less frequent than rheumatoid arthritis and not detected with cardiovascular risk indices. Arthritis Care Res (Hokoben) 68: 1538-1546.

7. Washington CW, Zipfel GJ (2011) Detection and monitoring of vasospasm and delayed cerebral ischemia: a review and assessment of the literature. Neurocrit Care 15: 312-317.

8. de Riva N, Budohoski KP, Smielewski P, Kasprowicz M, Zweifel C, et al. (2012) Transcranial Doppler Pulsatility Index: what it is and what it isn't. Neurocrit Care 17: 58-66.

9. Bos MJ, Koudstaal PJ, Hofman A, Witteman JC, Breteler MM (2007) Transcranial Doppler hemodynamic parameters and risk of stroke. The Rotterdam study. Stroke 38: 2453-2458. 